\title{
The entropic origin of disassortativity in complex networks
}

\author{
Samuel Johnson, Joaquín J. Torres, J. Marro, and Miguel A. Muñoz \\ Departamento de Electromagnetismo y Física de la Materia, and \\ Institute Carlos I for Theoretical and Computational Physics, \\ Facultad de Ciencias, University of Granada, 18071 Granada, Spain.
}

\begin{abstract}
Why are most empirical networks, with the prominent exception of social ones, generically degreedegree anticorrelated, i.e. disassortative? With a view to answering this long-standing question, we define a general class of degree-degree correlated networks and obtain the associated Shannon entropy as a function of parameters. It turns out that the maximum entropy does not typically correspond to uncorrelated networks, but to either assortative (correlated) or disassortative (anticorrelated) ones. More specifically, for highly heterogeneous (scale-free) networks, the maximum entropy principle usually leads to disassortativity, providing a parsimonious explanation to the question above. Furthermore, by comparing the correlations measured in some real-world networks with those yielding maximum entropy for the same degree sequence, we find a remarkable agreement in various cases. Our approach provides a neutral model from which, in the absence of further knowledge regarding network evolution, one can obtain the expected value of correlations. In cases in which empirical observations deviate from the neutral predictions - as happens in social networks - one can then infer that there are specific correlating mechanisms at work.
\end{abstract}

PACS numbers: 89.75.Fb, 89.75.Hc, 05.90.+m

Complex networks, whether natural or artificial, have non-trivial topologies which are usually studied by analysing a variety of measures, such as the degree distribution, clustering, average paths, modularity, etc. [13] The mechanisms which lead to a particular structure and their relation to functional constraints are often not clear and constitute the subject of much debate [2, 3]. When nodes are endowed with some additional "property," a feature known as mixing or assortativity can arise, whereby edges are not placed between nodes completely at random, but depending in some way on the property in question. If similar (dissimilar) nodes tend to wire together, the network is said to be assortative (disassortative) [4].

An interesting situation is when the property taken into account is the degree of each node - i.e., the number of neighboring nodes connected to it. It turns out that a high proportion of empirical networks - whether biological, technological, information-related or linguistic - are disassortatively arranged (high-degree nodes, or hubs, are preferentially linked to low-degree neighbors, and viceversa) while social networks are usually assortative. Such degree-degree correlations have important consequences for network characteristics such as connectedness and robustness [4].

However, while assortativity in social networks can be explained taking into account homophily [4] or modularity [5], the widespread prevalence and extent of disassortative mixing in most other networks remains somewhat mysterious. Maslov et al. found that the restriction of having at most one edge per pair of nodes induces some disassortative correlations in heterogeneous networks [6], and Park and Newman showed how this analogue of the Pauli exclusion principle leads to the edges following
Fermi statistics [7] (see also [8]). However, this restriction is not sufficient to fully account for empirical data. In general, when one attempts to consider computationally all the networks with the same distribution as a given empirical one, the mean assortativity is not necessarily zero [9]. But since some "randomization" mechanisms induce positive correlations and others negative ones [10], it is not clear how the phase space can be properly sampled numerically.

In this letter, we show that there is a general reason, consistent with empirical data, for the "natural" mixing of most networks to be disassortative. Using an information-theory approach we find that the configuration which can be expected to come about in the absence of specific additional constraints turns out not to be, in general, uncorrelated. In fact, for highly heterogeneous degree distributions such as those of the ubiquitous scale-free networks, we show that the expected value of the mixing is usually disassortative: there are simply more possible disassortative configurations than assortative ones. This result provides a simple topological answer to a long-standing question. Let us caution that this does not imply that all scale-free networks are disassortative, but only that, in the absence of further information on the mechanisms behind their evolution, this is the neutral expectation.

The topology of a network is entirely described by its adjacency matrix $\hat{a}$; the element $\hat{a}_{i j}$ represents the number of edges linking node $i$ to node $j$ (for undirected networks, $\hat{a}$ is symmetric). Among all the possible microscopically distinguishable configurations a set of $L$ edges can adopt when distributed among $N$ nodes, it is often convenient to consider the set of configurations which have certain features in common - typically some macro- 
scopic magnitude, like the degree distribution. Such a set of configurations defines an ensemble. In a seminal series of papers Bianconi has determined the partition functions of various ensembles of random networks and derived their statistical-mechanics entropy [11]. This allows the author to estimate the probability that a random network with certain constraints has of belonging to a particular ensemble, and thus assess the relative importance of different magnitudes and help discern the mechanisms responsible for a given real-world network. For instance, she shows that scale-free networks arise naturally when the total entropy is restricted to a small finite value. Here we take a similar approach: we obtain the Shannon information entropy encoded in the distribution of edges. As we shall see, both methods yield the same results [12], but for our purposes the Shannon entropy is more tractable.

The Shannon entropy associated with a probability distribution $p_{m}$ is $s=-\sum_{m} p_{m} \ln \left(p_{m}\right)$, where the sum extends over all possible outcomes $m$. For a given pair of nodes $(i, j), p_{m}$ can be considered to represent the probability of there being $m$ edges between $i$ and $j$. For simplicity, we shall focus here on networks such that $\hat{a}_{i j}$ can only take values 0 or 1 , although the method is applicable to any number of edges allowed. In this case, we have only two terms: $p_{1}=\hat{\epsilon}_{i j}$ and $p_{0}=1-\hat{\epsilon}_{i j}$, where $\hat{\epsilon}_{i j} \equiv E\left(\hat{a}_{i j}\right)$ is the expected value of the element $\hat{a}_{i j}$ given that the network belongs to the ensemble of interest. The entropy associated with pair $(i, j)$ is then $s_{i j}=-\left[\hat{\epsilon}_{i j} \ln \left(\hat{\epsilon}_{i j}\right)+\left(1-\hat{\epsilon}_{i j}\right) \ln \left(1-\hat{\epsilon}_{i j}\right)\right]$, while the total entropy of the network is $S=\sum_{i j}^{N} s_{i j}$ :

$$
S=-\sum_{i j}^{N}\left[\hat{\epsilon}_{i j} \ln \left(\hat{\epsilon}_{i j}\right)+\left(1-\hat{\epsilon}_{i j}\right) \ln \left(1-\hat{\epsilon}_{i j}\right)\right] .
$$

Since we have not imposed symmetry of the adjacency matrix, this expression is in general valid for directed networks. For undirected networks, however, the sum is only over $i \leq j$, with the consequent reduction in entropy.

For the sake of illustration, we shall estimate the entropy of the Internet at the autonomous system (AS) level and compare it with the values obtained in [11] assuming the network belongs to two different ensembles: the fully random graph, or Erdös-Rényi (ER) ensemble, and the configuration ensemble with a scalefree degree distribution $\left(p(k) \sim k^{-\gamma}\right)$ [2] and structural cutoff, $k_{i}<\sqrt{\langle k\rangle N}, \forall i[11]$ ( $\langle k\rangle$ is the mean degree). In this example, we assume the network to be sparse enough to expand the term $\ln \left(1-\hat{\epsilon}_{i j}\right)$ in Eq. (11) and keep only linear terms. This reduces Eq. (1) to $S_{\text {sparse }} \simeq-\sum_{i j}^{N} \hat{\epsilon}_{i j}\left[\ln \left(\hat{\epsilon}_{i j}\right)-1\right]+O\left(\hat{\epsilon}_{i j}^{2}\right)$. In the ER ensemble, each of $N$ nodes has an equal probability of receiving each of $\frac{1}{2}\langle k\rangle N$ undirected edges. So, writing $\hat{\epsilon}_{i j}^{E R}=\langle k\rangle / N$, we have $S_{E R}=-\frac{1}{2}\langle k\rangle N[\ln (\langle k\rangle / N)-1]$. The configuration ensemble, which imposes a given degree sequence $\left(k_{1}, \ldots k_{N}\right)$, is defined via the expected value of the adjacency matrix: $\hat{\epsilon}_{i j}^{c}=k_{i} k_{j} /(\langle k\rangle N)$ [2, 13]. This value leads to $S_{c}=\langle k\rangle N[\ln (\langle k\rangle N)+1]-2 N\langle k \ln k\rangle$, where $\langle\cdot\rangle \equiv N^{-1} \sum_{i}(\cdot)$ stands for an average over nodes.

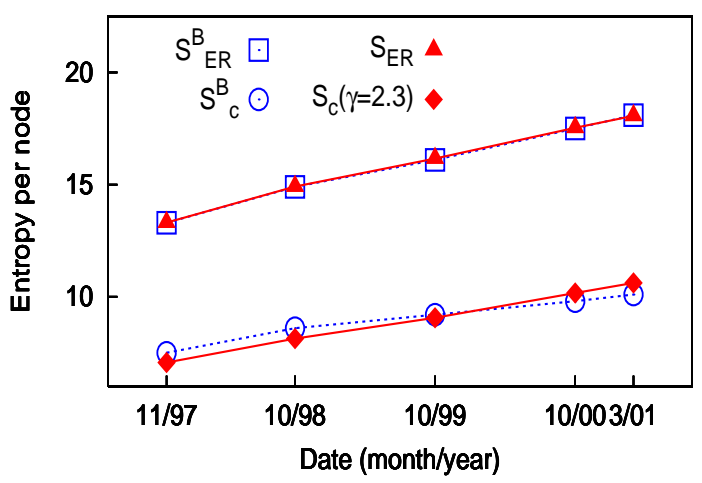

FIG. 1: (Color online) Evolution of the Internet at the AS level. Empty (blue) squares and circles: entropy per node of randomized networks in the fully random and in the configuration ensembles, as obtained by Bianconi (hence the "B" superscription) [1]. Filled (red) triangles and diamonds: Shannon entropy for an ER network and a scale-free one with $\gamma=2.3$, respectively.

Fig. 1 displays the entropy per node obtained in [11] for the first two levels of approximation (ensembles) to the Internet at the AS level, first taking into account only the numbers of nodes $N$ and edges $L=\frac{1}{2}\langle k\rangle N$, and then also the degree sequence. Alongside these, we plot the Shannon entropy both for an ER random network, (which coincides exactly with Bianconi's expression), and for a scale-free network with $\gamma=2.3$ (the slight disparity arising from this exponent's changing a little with time).

We shall now go on to analyse the effect of degreedegree correlations on the entropy. In the configuration ensemble, the expected value of the mean degree of the neighbors of a given node is $k_{n n, i}=k_{i}^{-1} \sum_{j} \hat{\epsilon}_{i j}^{c} k_{j}=$ $\left\langle k^{2}\right\rangle /\langle k\rangle$, which is independent of $k_{i}$. However, as mentioned above, real networks often display degree-degree correlations, with the result that $k_{n n, i}=k_{n n}\left(k_{i}\right)$. If $k_{n n}(k)$ increases (decreases) with $k$, the network is assortative (disassortative). A measure of this phenomenon is Pearson's coefficient applied to the edges [2-4] : $r=$ $\left(\left[k_{l} k_{l}^{\prime}\right]-\left[k_{l}\right]^{2}\right) /\left(\left[k_{l}^{2}\right]-\left[k_{l}\right]^{2}\right)$, where $k_{l}$ and $k_{l}^{\prime}$ are the degrees of each of the two nodes belonging to edge $l$, and $[\cdot] \equiv(\langle k\rangle N)^{-1} \sum_{l}(\cdot)$ is an average over edges. Writing $\sum_{l}(\cdot)=\sum_{i j} \hat{a}_{i j}(\cdot), r$ can be expressed as

$$
r=\frac{\langle k\rangle\left\langle k^{2} k_{n n}(k)\right\rangle-\left\langle k^{2}\right\rangle^{2}}{\langle k\rangle\left\langle k^{3}\right\rangle-\left\langle k^{2}\right\rangle^{2}} .
$$

The ensemble of all networks with a given degree sequence $\left(k_{1}, \ldots k_{N}\right)$ contains a subset for all members of which $k_{n n}(k)$ is constant (the configuration ensemble), but also subsets displaying other functions $k_{n n}(k)$. We 

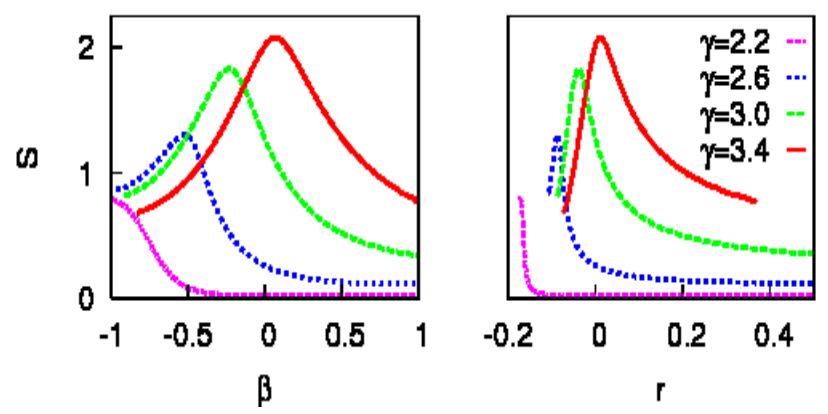

FIG. 2: (Color online) Shannon entropy of correlated scalefree networks against parameter $\beta$ (left panel) and against Pearson's coefficient $r$ (right panel), for various values of $\gamma$ (increasing from bottom to top). $\langle k\rangle=10, N=10^{4}$.

can identify each one of these subsets (regions of phase space) with an expected adjacency matrix $\hat{\epsilon}$ which simultaneously satisfies the following conditions: i) $\sum_{j} k_{j} \hat{\epsilon}_{i j}=$ $k_{i} k_{n n}\left(k_{i}\right), \forall i$, and ii) $\sum_{j} \hat{\epsilon}_{i j}=k_{i}, \forall i$ (for consistency). An ansatz which fulfills these requirements is any matrix of the form

$$
\hat{\epsilon}_{i j}=\frac{k_{i} k_{j}}{\langle k\rangle N}+\int d \nu \frac{f(\nu)}{N}\left[\frac{\left(k_{i} k_{j}\right)^{\nu}}{\left\langle k^{\nu}\right\rangle}-k_{i}^{\nu}-k_{j}^{\nu}+\left\langle k^{\nu}\right\rangle\right],
$$

where $\nu \in \mathbb{R}$ and the function $f(\nu)$ is in general arbitrary, although depending on the degree sequence it shall here be restricted to values which maintain $\hat{\epsilon}_{i j} \in[0,1], \forall i, j$. This ansatz yields

$$
k_{n n}(k)=\frac{\left\langle k^{2}\right\rangle}{\langle k\rangle}+\int d \nu f(\nu) \sigma_{\nu+1}\left[\frac{k^{\nu-1}}{\left\langle k^{\nu}\right\rangle}-\frac{1}{k}\right]
$$

(the first term being the result for the configuration ensemble), where $\sigma_{b+1} \equiv\left\langle k^{b+1}\right\rangle-\langle k\rangle\left\langle k^{b}\right\rangle$. In practice, one could adjust Eq. (44) to fit any given function $k_{n n}(k)$ and then wire up a network with the desired correlations: it suffices to throw random numbers according to Eq. (3) with $f(\nu)$ as obtained from the fit to Eq. (4) [14]. To prove the uniqueness of a matrix $\hat{\epsilon}$ obtained in this way (i.e., that it is the only one compatible with a given $\left.k_{n n}(k)\right)$ assume that there exists another valid matrix $\hat{\epsilon}^{\prime} \neq \hat{\epsilon}$. Writting $\hat{\epsilon}_{i j}^{\prime}-\hat{\epsilon}_{i j} \equiv h\left(k_{i}, k_{j}\right)=h_{i j}$, then i) implies that $\sum_{j} k_{j} h_{i j}=0, \forall i$, while ii) means that $\sum_{j} h_{i j}=0, \forall i$. It follows that $h_{i j}=0, \forall j$.

In many empirical networks, $k_{n n}(k)$ has the form $k_{n n}(k)=A+B k^{\beta}$, with $A, B>0[3,15]$ - the mixing being assortative (disassortative) if $\beta$ is positive (negative). Such a case is fitted by Eq. (4) if $f(\nu)=$ $C\left[\delta(\nu-\beta-1) \sigma_{2} / \sigma_{\beta+2}-\delta(\nu-1)\right]$, with $C$ a positive constant, since this choice yields

$$
k_{n n}(k)=\frac{\left\langle k^{2}\right\rangle}{\langle k\rangle}+C \sigma_{2}\left[\frac{k^{\beta}}{\left\langle k^{\beta+1}\right\rangle}-\frac{1}{\langle k\rangle}\right] .
$$

After plugging Eq. (5) into Eq. (2), one obtains:

$$
r=\frac{C \sigma_{2}}{\left\langle k^{\beta+1}\right\rangle}\left(\frac{\langle k\rangle\left\langle k^{\beta+2}\right\rangle-\left\langle k^{2}\right\rangle\left\langle k^{\beta+1}\right\rangle}{\langle k\rangle\left\langle k^{3}\right\rangle-\left\langle k^{2}\right\rangle^{2}}\right) .
$$

Inserting Eq. (3) in Eq. (11), we can calculate the entropy of correlated networks as a function of $\beta$ and $C-$ or, by using Eq. (6), as a function of $r$. Particularizing for scale-free networks, then given $\langle k\rangle, N$ and $\gamma$, there is always a certain combination of parameters $\beta$ and $C$ which maximizes the entropy; we shall call these $\beta^{*}$ and $C^{*}$. For $\gamma \lesssim 5 / 2$ this point corresponds to $C^{*}=1$. For higher $\gamma$, the entropy can be slightly higher for larger $C$. However, for these values of $\gamma$, the assortativity $r$ of the point of maximum entropy obtained with $C=1$ differs very little from the one corresponding to $\beta^{*}$ and $C^{*}$ (data not shown). Therefore, for the sake of clarity but with very little loss of accuracy, in the following we shall generically set $C=1$ and vary only $\beta$ in our search for the level of assortativity, $r^{*}$, that maximizes the entropy given $\langle k\rangle, N$ and $\gamma$. Note that $C=1$ corresponds to removing the linear term, proportional to $k_{i} k_{j}$, in Eq. (3), and leaving the leading non-linearity, $\left(k_{i} k_{j}\right)^{\beta+1}$, as the dominant one.

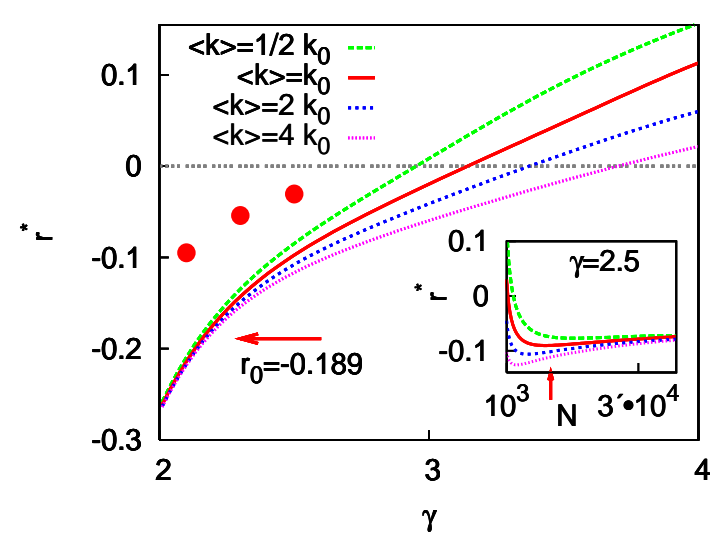

FIG. 3: (Color online) Lines from top to bottom: $r$ at which the entropy is maximized, $r^{*}$, against $\gamma$ for random scale-free networks with mean degrees $\langle k\rangle=\frac{1}{2}, 1,2$ and 4 times $k_{0}=$ 5.981 , and $N=N_{0}=10697$ nodes $\left(k_{0}\right.$ and $N_{0}$ correspond to the values for the Internet at the AS level in 2001 [7], which had $\left.r=r_{0}=-0.189\right)$. Symbols are the values obtained in [7] as those expected solely due to the one-edge-per-pair restriction (with $k_{0}, N_{0}$ and $\gamma=2.1,2.3$ and 2.5). Inset: $r^{*}$ against $N$ for networks with fixed $\langle k\rangle / N$ (same values as the main panel) and $\gamma=2.5$; the arrow indicates $N=N_{0}$.

Fig. 2 displays the entropy curves for various scalefree networks, both as functions of $\beta$ and of $r$ : depending on the value of $\gamma$, the point of maximum entropy can be either assortative or disassortative. This can be seen more clearly in Fig. 3, where $r^{*}$ is plotted against $\gamma$ for scale-free networks with various mean degrees $\langle k\rangle$. The values obtained by Park and Newman [7] as those 
resulting from the one-edge-per-pair restriction are also shown for comparison: notice that whereas this effect alone cannot account for the Internet's correlations for any $\gamma$, entropy considerations would suffice if $\gamma \simeq 2.1$. As shown in the inset, the results are robust in the large system-size limit (although see [16]).

Since most networks observed in the real world are highly heterogeneous, with exponents in the range $\gamma \in$ $(2,3)$, it is to be expected that these should display a certain disassortativity - the more so the lower $\gamma$ and the higher $\langle k\rangle$. In Fig. 4 we test this prediction on a sample of empirical, scale-free networks quoted in Newman's review [2] (p. 182). For each case, we found the value of $r$ that maximizes $S$ according to Eq. (11), after inserting Eq. (3) with the quoted values of $\langle k\rangle, N$ and $\gamma$. In this way, we obtained the expected assortativity for six networks, representing: a peer-to-peer (P2P) network, metabolic reactions, the nd.edu domain, actor collaborations, protein interactions, and the Internet (see [2] and references therein). For the metabolic, Web domain and protein networks, the values predicted are in excellent agreement with the measured ones; therefore, no specific anticorrelating mechanisms need to be invoked to account for their disassortativity. In the other three cases, however, the predictions are not accurate, so there must be additional correlating mechanisms at work. Indeed, it is known that small routers tend to connect to large ones [15], so one would expect the Internet to be more disassortative than predicted, as is the case [17] an effect that is less pronounced but still detectable in the more egalitarian P2P network. Finally, as is typical of social networks, the actor graph is significantly more assortative than predicted, probably due to the homophily mechanism whereby highly connected, big-name actors tend to work together [4].

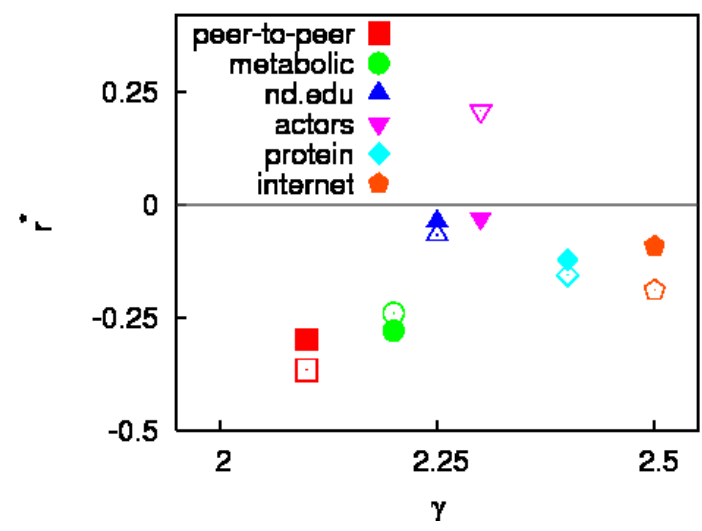

FIG. 4: (Color online) Level of assortativity that maximizes the entropy, $r^{*}$, for various real-world, scale-free networks, as predicted theoretically by Eq. (1) (solid symbols) and as directly measured (empty symbols), against exponent $\gamma$.

In summary, we have shown how the ensemble of net- works with a given degree sequence can be partitioned into regions of equally correlated networks and found, using an information-theory approach, that the largest (maximum entropy) region, for the case of scale-free networks, usually displays a certain disassortativity. Therefore, in the absence of knowledge regarding the specific evolutionary forces at work, this should be considered the most likely state. Given the accuracy with which our approach can predict the degree of assortativity of certain empirical networks with no a priori information thereon, we suggest this as a neutral model to decide whether or not particular experimental data require specific mechanisms to account for observed degree-degree correlations.

This work was supported by the Junta de Andalucía project P09-FQM4682, and by Spanish MICINNFEDER project FIS2009-08451.

[1] R. Albert A.-L. and Barabási, Rev. Mod. Phys. 74, 47 (2002) S.N. Dorogovtsev and J.F.F. Mendes, Evolution of networks: From biological nets to the Internet and $W W W$, Oxford Univ, Press, 2003. R. Pastor-Satorras and A. Vespignani, Evolution and structure of the Internet: A statistical physics approach, Cambridge Univ. Press, Cambridge, 2004.

[2] M.E.J. Newman, SIAM Reviews 45, 167 (2003).

[3] S. Boccaletti, V. Latora, Y. Moreno, M. Chavez, and D.U. Hwang, Phys. Rep. 424, 175 (2006).

[4] M.E.J. Newman, Phys. Rev. Lett., 89, 208701 (2002); Phys. Rev. E 67, 026126 (2003).

[5] M.E.J. Newman and J. Park, Phys. Rev. E 68, 036122 (2003).

[6] S. Maslov, K. Sneppen, and A. Zaliznyak, Physica A 333, 529-540 (2004).

[7] J. Park and M.E.J. Newman, Phys. Rev. E 68, 026112 (2003).

[8] A. Capocci and F. Colaiori, Phys. Rev. E. 74, 026122 (2006).

[9] P. Holme and J. Zhao Phys. Rev. E 75, 046111 (2007).

[10] I. Farkas, I. Derényi, G. Palla, and T. Vicsek, Lect. Notes in Phys. 650, 163 (2004). S. Johnson, J. Marro, and J.J. Torres, J. Stat. Mech., in press. arXiv: 0905.3759.

[11] G. Bianconi, EPL 81, 28005 (2007); Phys. Rev. E 79, 036114 (2009).

[12] E.T. Jaynes, Physical Review 106, 620-630 (1957). K. Anand and G. Bianconi, Phys. Rev. E 80 045102(R) (2009).

[13] S. Johnson, J. Marro, and J.J. Torres, EPL 83, 46006 (2008).

[14] Although, as with the configuration ensemble, it is not always possible to wire a network according to a given $\hat{\epsilon}$.

[15] R. Pastor-Satorras, A. Vázquez, and A. Vespignani, Phys. Rev. Lett. 87, 258701 (2001).

[16] S.N. Dorogovtsev, A.L. Ferreira, A.V. Goltsev, and J.F.F. Mendes. arXiv: 0911.4285v1.

[17] However, as Fig. 3 shows, if the Internet exponent were the $\gamma=2.2 \pm 0.1$ reported in [15] rather than $\gamma=2.5$, entropy would account more fully for these correlations. 